\title{
Measuring the degree of stacking order in graphite by Raman spectroscopy
}

\author{
L.G. Cançado, K. Takai, and T. Enoki \\ Department of Chemistry, Tokyo Institute of Thecnology, \\ 2-12-1, Ookayama, Meguro-ku, Tokyo 152-8551, Japan.
}

M. Endo, Y. A. Kim, and H. Mizusaki

Faculty of Engeneering, Shinshu University 4-17-1 Wakasato, Nagano-shi 380-8553, Japan.

A. Jorio, N. L. Speziali, and M. A. Pimenta

Departamento de Física, Universidade Federal de Minas Gerais, 30123-970, Belo Horizonte, Brazil.

(Dated: Submitted on June 13, 2007)

\begin{abstract}
This work reports the analysis of the $G^{\prime}$ band profile in the Raman spectra of nanographites with different degrees of stacking order. Since the $G^{\prime}$ band scattering coming from the 2D and 3D phases coexisting in the same sample can be nicely distinguished, the relative volumes of 3D and 2D graphite phases present in the samples can be estimated from their Raman spectra. The comparison between Raman scattering and X-Ray diffraction data shows that Raman spectroscopy can be used as an alternative tool for measuring the degree of stacking order of graphitic systems.
\end{abstract}

\section{INTRODUCTION}

Raman spectroscopy plays an important role in the structural characterization of graphitic materials. The ratio between the intensities of the disorder-induced $D$ band (at $\sim 1350 \mathrm{~cm}^{-1}$ ) and the first-order allowed $G$ band (at $\sim 1580 \mathrm{~cm}^{-1}$ ) $I_{D} / I_{G}$ is inversely proportional to the in-plane crystallite size $L_{a} \cdot{ }^{1-4}$ On the other hand, the second harmonic of the $D$ band, the $G^{\prime}$ band (at $\sim 2700 \mathrm{~cm}^{-1}$ ) is very sensitive to structural changes along the $c$ axes, since its profile changes from a single peak to two peaks in the Raman spectra obtained from turbostratic to crystalline graphite. ${ }^{5-7}$ Wilhelm et al. suggested that the origin of the double structure of the $G^{\prime}$ band in crystalline graphite was associated with the stacking order occurring along the $c$ axes. ${ }^{8}$ Recent works have reported the evolution of the $G^{\prime}$ band in the Raman spectra obtained from one to a multilayer graphene structure. ${ }^{9-11}$ These works show that the $G^{\prime}$ band of a monolayer graphene is composed by a single peak, whereas a two-peaks profile is observed for samples formed by a large number of graphene layers, giving the definite proof for the hypothesis of Wilhelm et al. ${ }^{8}$

This manuscript reports a study of Raman scattering in nanographite samples with different degrees of graphitization. It will be shown that the changes in the $G^{\prime}$ band from a one-peak to a two-peak profile allow us to distinguish the relative volumes of the $3 \mathrm{D}$ and $2 \mathrm{D}$ graphitic phases coexisting in the same sample. The comparison between the Raman scattering and X-Ray diffraction data shows that the out-of plane lattice parameter $c$ and the crystallite thickness $L_{c}$ can be quantitatively determined from the ratio between the $G^{\prime}$ band scattering intensities obtained from the $2 \mathrm{D}$ and $3 \mathrm{D}$ graphite phases.

\section{EXPERIMENTAL DETAILS}

The samples used in the experiment are disordered graphite films heat-treated at different temperatures, giving rise to nanographites with different crystallinity degrees. The films were prepared by a pulsed laser deposition method. The heat-treatment was made using an electrical furnace setup, at heat-treatment temperatures (HTT) of $2200^{\circ} \mathrm{C}, 2300^{\circ} \mathrm{C}, 2500^{\circ} \mathrm{C}$ and $2700^{\circ} \mathrm{C}$ (see Reference [4] for details). Raman scattering measurements were performed using a triple monochromator microRaman spectrometer (DILOR XY) using the following laser wavelengths (energies): Krypton $647 \mathrm{~nm}(1.92 \mathrm{eV})$ and $568 \mathrm{~nm}(2.18 \mathrm{eV})$, and Argon $514.5 \mathrm{~nm}(2.41 \mathrm{eV})$, $488 \mathrm{~nm}(2.54 \mathrm{eV})$ and $457.9 \mathrm{~nm}(2.71 \mathrm{eV}) .{ }^{19}$ X-ray diffraction measurements where performed using a Rigaku setup, in $(\theta / 2 \theta)$ geometry, using a copper X-ray emission tube.

\section{RESULTS AND DISCUSSION}

Figure 1 shows the $\mathrm{G}^{\prime}$ band spectra of partially disordered graphite samples heat-treated at different temperatures, obtained using the $514.5 \mathrm{~nm}$ excitation laser wavelength. The Raman spectrum of the sample heattreated at $2200^{\circ} \mathrm{C}$ (bottom part) can be fit using only one single peak centered at $2707 \mathrm{~cm}^{-1}$, called here $G_{2 D}^{\prime}$. This is the typical profile of the $G^{\prime}$ band in Raman spectra of two-dimensional graphite samples..$^{8-11}$ At the top, the spectrum of the sample heat-treated at $2700^{\circ} \mathrm{C}$ presents a two-peaks shape, which is the typical profile for the $G^{\prime}$ band in 3D graphite samples. ${ }^{8-11}$ Notice that this band can be fit using two Lorentzians $\left(\mathrm{G}_{3 D A}^{\prime}\right.$ and $\left.G_{3 D B}^{\prime}\right)$ centered at $2687 \mathrm{~cm}^{-1}$ and $2727 \mathrm{~cm}^{-1}$ respectively. The Raman spectra of the samples heat-treated at intermediate temperature values $\left(2300^{\circ} \mathrm{C}\right.$, and $\left.2500^{\circ} \mathrm{C}\right)$ show the evolution of the $G^{\prime}$ band from a one-peak to a two-peak 


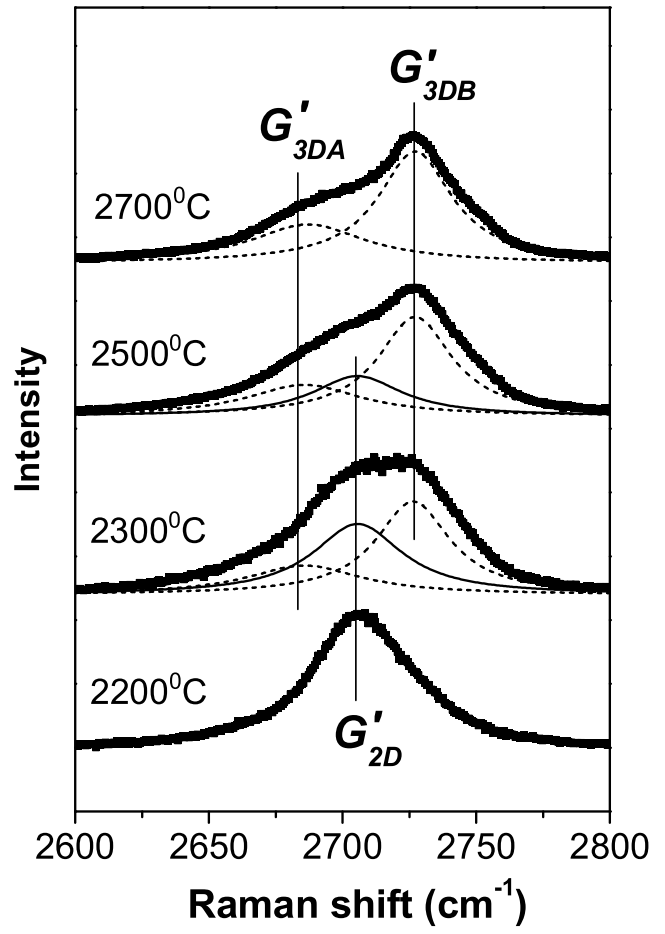

FIG. 1: $G^{\prime}$ band Raman spectra of partially disordered graphite samples heat-treated at different temperatures, performed using the excitation laser wavelength of $514.5 \mathrm{~nm}$ $(2.41 \mathrm{eV})$. The $\mathrm{G}^{\prime}$ band changes from a one-peak to a twopeak profile with increasing heat-treatment temperature.

profile. Three Lorentzian peaks are needed to fit the Raman spectra of these samples. The relative intensity (integrated area) of the $G_{3 D A}^{\prime}$ and $G_{3 D B}^{\prime}$ peaks $\left(I_{G_{3 D A}^{\prime}}\right.$ and $I_{G_{3 D B}^{\prime}}$ respectively) increases while the relative intensity of the $G_{2 D}^{\prime}$ peak $\left(I_{G_{2 D}^{\prime}}\right)$ decreases with increasing heat-treatment temperature. Furthermore, for all spectra depicted in Fig. 1, the intensity ratio of the peaks $G_{3 D A}^{\prime}$ and $G_{3 D B}^{\prime}$ is constant, being $I_{G_{3 D B}^{\prime}} / I_{G_{3 D A}^{\prime}} \sim 2$. The same result was obtained for the other four excitation laser wavelengths used in the experiment (not shown in Fig. 1).

The two-peak profile of the $G^{\prime}$ band in the Raman spectrum obtained from the sample heat-treated at $2700^{\circ} \mathrm{C}$ is caused by a splitting in the $\pi$ electrons dispersion occurring for the $3 \mathrm{D}$ graphite lattice. ${ }^{9}$ For the sample heattreated at $2200^{\circ} \mathrm{C}$, the $G^{\prime}$ band is composed by a single peak, indicating that the interaction between the basal planes is weak enough so that the splitting in the $\pi$ electrons dispersion energies does not occurs, being the sample composed by a turbostratic structure.

The coexistence of the doublet $G_{3 D A}^{\prime}$ and $G_{3 D B}^{\prime}$ with the $G_{2 D}^{\prime}$ peak in the Raman spectra of the samples heattreated at $2300^{\circ} \mathrm{C}$ and $2500^{\circ} \mathrm{C}$ indicates the simultaneous presence of the $3 \mathrm{D}$ and $2 \mathrm{D}$ graphite phases in these

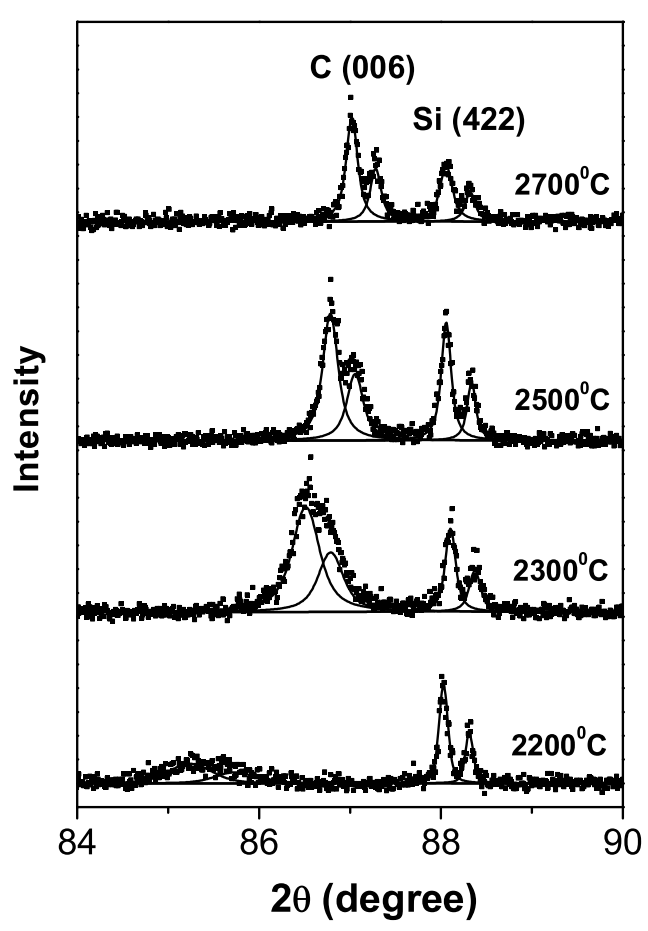

FIG. 2: X-ray diffraction profile of the (006) peak of the heat-treated samples. The heat-treatment temperature is indicated at the right side of the respective curve.

samples. Considering $V$ as the volume of the sample which is illuminated by the incident laser beam, there is a fraction of $V$ composed by only the 3D graphite phase $\left(V_{3 D}\right)$, and another one $\left(V_{2 D}\right)$ composed by the turbostratic graphite, being $V=V_{3 D}+V_{2 D}$. Since the Raman intensity is proportional to the volume of the sample illuminated from the incident laser beam, and the contribution to the $G^{\prime}$ band from the $3 \mathrm{D}$ and $2 \mathrm{D}$ phases coexisting in the same sample can be distinguished, ${ }^{12}$ the relative volumes $v_{3 D}=V_{3 D} / V$ and $v_{2 D}=V_{2 D} / V$ can be estimated from the ratio $R$ given by:

$$
R=\left|\frac{I_{G_{3 D B}^{\prime}}}{I_{G_{3 D B}^{\prime}}+I_{G_{2 D}^{\prime}}}\right|,
$$

being $v_{3 D}=R$, and $v_{2 D}=1-R$.

In an early work, Franklin has shown that, in a sample were the $2 \mathrm{D}$ and $3 \mathrm{D}$ graphitic phases coexist, the intermediate value of the out-of-plane lattice parameters measured from the X-Ray diffraction profiles is, in fact, an average value determined by the relative amount of the $2 \mathrm{D}$ and $3 \mathrm{D}$ phases composing the sample. ${ }^{13}$ In this case, since the Raman spectrum of such samples can estimate the relative volumes of the $2 \mathrm{D}$ and $3 \mathrm{D}$ phases, the average value of the lattice parameter can be obtained from the ratio $R$. 


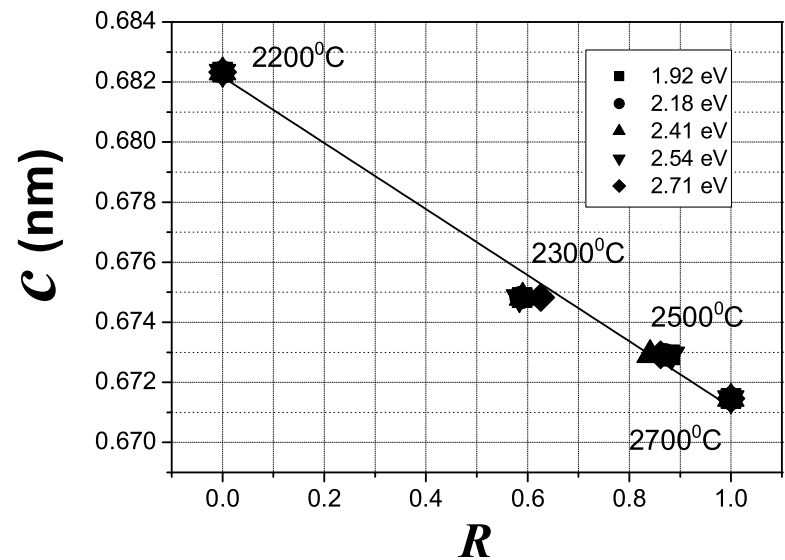

FIG. 3: Plot of the average out-of-plane lattice parameter $c=6 \cdot d_{(006)}$ of the heat-treated samples obtained from the $\mathrm{X}$-ray diffraction data depicted in Figure 2 s the ratio $R$ for the five excitation laser energies used in the experiment. The solid line is the linear fit giving according to Equation 2.

In order to check this assumption, we performed an $\mathrm{X}$-ray diffraction analysis of the samples. Figure 2 shows the X-ray diffraction profile of the (006) peak of the samples heat-treated at different temperatures. The profiles are composed of two peaks, related to the $\kappa_{\alpha_{1}}$ and $\kappa_{\alpha_{2}}$ lines from the copper X-ray tube emission. From the Xray diffraction peaks, the interlayer spacing $\left[d_{(006)}\right]$ can be obtained as $d_{(006)}=\lambda / 2 \sin \theta$, where $\lambda$ is the wavelength of the copper $\kappa_{\alpha_{1}}$ X-ray line $(\lambda=0.154 \mathrm{~nm})$, and $\theta$ is the diffraction angle of the (006) peak. ${ }^{14}$ To avoid the intrinsic instrumental error, the diffraction angle $\theta$ was corrected from the value of the diffraction angle of the (422) peak of the standard silicon sample (shown in Figure 2). Figure 3 shows the plot of the average out-of-plane lattice parameter $c=6 \cdot d_{(006)}$ of the heattreated samples obtained from the X-ray diffraction data depicted in Figure $2 v s$ the ratio $R$ for the five excitation laser energies. It is clear in Fig. 3 that there is a linear dependence between the parameters $R$ and $c$. The solid line in Fig. 3 is the linear linear fit of the data given by:

$$
c(n m)=0.682-0.11 R .
$$

Equation 2 provides a formula which allow us to evaluate the out-of-plane lattice parameter of partially disordered graphites from Raman scattering experiments using any excitation laser energy in the visible range.

The value for the out-of-plane lattice parameter obtained for the sample heat-treated at $2700^{\circ} \mathrm{C}$ $(c=0.671 \mathrm{~nm})$ is in excellent agreement with the value of $c$ for crystalline graphite. ${ }^{13}$ However, the value of the outof-plane lattice parameter obtained here for the sample heat-treated at $2200^{\circ} \mathrm{C}(c=0.682 \mathrm{~nm})$ is lower than that established for turbostratic samples $(c=0.688 \mathrm{~nm}) .^{13}$
In fact, for samples with values of $c$ ranging around $0.688 \mathrm{~nm}$, Babu and Seehra have pointed out that these systems are no longer purely graphitic. ${ }^{15}$ Instead, these samples are formed not only by $s p^{2}$ bonds, but also by $s p^{3}$, which presence causes a considerable expansion in the interlayer distance due to lattice deformations. ${ }^{16}$ As previously reported by Takai et al. ${ }^{16}$ the nanographite samples used here do not contain $s p^{3}$ bounds, explaining why this relatively high value for the out-of-plane lattice parameter was not detected in our X-Ray analysis. This conclusion is also supported by the fact that the $G_{2 D}^{\prime}$ peak present in the Raman spectrum of the sample heattreated at $2200^{\circ} \mathrm{C}$ shown in Fig. 1 has a considerable narrow linewidth if compared with samples composed by amorphous carbon (see for example Reference [15]). This is an indication of a large in-plane phonon lifetime, occurring for purely graphitic samples with good in-plane crystallinity degree. ${ }^{17}$

Another important factor in the analysis of the stacking order of graphite is the crystallite thickness $L_{c}$. Raman spectroscopy is known to be an useful tool for measuring the in-plane crystallite size $L_{a}$ in nano-sized graphite materials. ${ }^{1-4}$ We show here that the dependence of the $G^{\prime}$ band profile of graphitic samples with different degrees of stacking order shown in Figure 1 can also give a quantitative information about their average crystallite thickness $L_{c}$. Figure 4 shows the plot of the $L_{c}$ values obtained from the X-ray data depicted in Figure 2 s s the ratio $R$ taken from the Raman spectra obtained for the five different excitation laser energies. The $L_{c}$ parameter was evaluated from the Scherrer equation $L_{c}=0.91 \lambda /(\beta \cdot \cos \theta)$, where $\beta$ is the half-height width of the (006) diffraction peak in $2 \theta$ (rad) units. ${ }^{14}$ To avoid the intrinsic instrumental broadening, the $\beta$ parameter was corrected using the equation $\beta=\sqrt{\beta_{m}^{2}-\beta_{S i}^{2}}$, where $\beta_{m}$ is the half-height width of the measured (006) peak of the samples, and $\beta_{S i}$ is the half-height width of the (422) peak of the standard silicon sample. The plot in Figure 4 shows that $L_{c}$ increases with increasing $R$ in the same way for the five excitation laser energies, indicating that the heat-treatment process performed in these samples increases the crystallite thickness $L_{c}$ which can be detected by the Raman spectra. The solid line is a fit of the experimental data giving the empirical formula:

$$
L_{c}(n m)=10+\frac{10}{1.05-0.05 R}
$$

relating the average crystallite thickness $L_{c}$ and the ratio $R$ for any excitation laser energy in the visible range. It should be emphasized here that although the parameters $c$ and $L_{c}$ can be obtained directly from the X-Ray diffraction, Equations 2 and 3 provide a valuable alternative way for measure them, since Raman spectroscopy is a versatile non-destructive technique for which special sample preparation procedures are not necessary, and the data acquisition time is relatively short. 


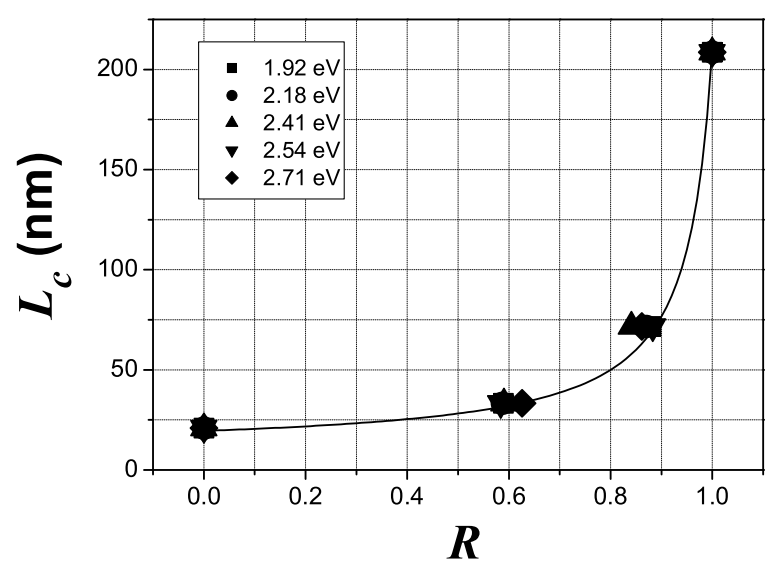

FIG. 4: Plot of the average out-of-plane crystallite thickness $L_{c}$ of the heat-treated samples obtained from the X-ray diffraction data depicted in Figure $2 v s$ the ratio $R$ for the five excitation laser energies used in the experiment.

\section{FINAL REMARKS}

In summary, this manuscript shows that the analysis of the $G^{\prime}$ band profile in the Raman spectra of partially disordered graphites give the information about the relative volumes of $3 \mathrm{D}$ and $2 \mathrm{D}$ graphite phases present in the samples. Since the average value of the lattice parameter $c$ is determined by the fraction of 3D and 2D phases coexisting in the same sample, we show that Raman spectroscopy can be used as an alternative tool for measuring the out-of-plane lattice parameter of nanographitic systems. An empirical formula relating the ratio $R$ obtained from Raman scattering and the crystallite thickness $L_{c}$ of nanographites is determined for any excitation laser energy used in the experiment.

\section{ACKNOWLEDGEMENTS}

This work was supported by the Brazilian Network on Carbon Nanotube Research-MCT and FAPEMIG. L.G.C. acknowledges the support from the Brazilian Agency CNPq, and from TIT during his visit to TIT. The authors acknowledge Alexandre de Melo Moreira for the technical support during the X-Ray diffraction measurements.

${ }^{1}$ F. Tuinstra and J. L. Koenig, J. Chem. Phys. 53, 1126 (1970).

${ }^{2}$ F. Tuinstra and J. L. Koenig, J. Composite Materials 4, 492 (1970).

3 D. S. Knight and W. White, J. Mater. Res. 4, 385 (1989).

${ }^{4}$ L.G. Cançado, K. Takai, T. Enoki, M. Endo, Y. A. Kim, H. Mizusaki, A. Jorio, L. N. Coelho, R. Magalhães-Paniago, and M. A.Pimenta, Appl. Phys. Letters 88, 163106 (2006).

${ }^{5}$ R. J. Nemanich and S. A. Solim, Solid State Comm. 23, 417 (1977).

${ }^{6}$ R. J. Nemanich and S. A. Solim, Phys. Rev. B 20, 392 (1979).

7 P. Lespade, A. Marchand, M. Couzi, and F. Cruege, Carbon 22, 375 (1984)

${ }^{8}$ H. Wilhelm, M. Lelaurain, McRae, and B. Humbert, Journal of Appl. Physics 84, 6552 (1998).

9 A. C. Ferrari, J. C. Meyer, V. Scardaci, C. Casiraghi, M. Lazzeri, F. Mauri, S. Piscanec, D. Jiang, K. S. Novoselov, S. Roth, and A. K. Geim, Phys. Rev. Lett. 97, 187401 (2006).
10 A. Gupta, G. Chen, P. Joshi, S. Tadigadapa, and P.C. Eklund, Nanoletters 6, 2667 (2006).

11 D. Graf, F. Molitor, K. Ensslin, C. Stampfer, A. Jungen, C. Hierold, and L. Wirtz, Nanoletters, (2007).

12 E. B. Barros, N. S. Demir, A. G. Souza-Filho, J. Mendes Filho, A. Jorio, G. Dresselhaus, and M. S. Dresselhaus, Phys. Rev. B 71, 165422 (2005).

13 Rosalind E. Franklin, Acta Crystal. 4, 253 (1951).

14 M. S. Seehra and A. S. Pavlovic, Carbon 31, 557 (1992).

15 V. Suresh Babu and M. S. Seehra, Carbon 34, 1259 (1996).

${ }^{16}$ K. Takai, M. Oga, H. Sato, T. Enoki, Y. Ohki, A. Taomoto, K. Suenaga, S. Iijima, Phys. Rev. B, 67, 214202 (2003).

17 L. G. Cançado, A. Jorio, and M. A. Pimenta, submmited.

18 L.G. Cançado, M. A. Pimenta, R. Saito, A. Jorio, L. O. Ladeira, A. Gruneis, A. G. Souza-Filho, G. Dresselhaus, and M. S. Dresselhaus, Phys. Rev. B 66, 035415 (2002).

19 It is important to characterize the $G^{\prime}$ band intensity behavior with different excitation laser lines, since the $G^{\prime}$ band scattering is known to exhibit a resonance behavior. ${ }^{18}$ 